\title{
Measuring variation for nominal data
}

\author{
TARALD O. KVÄLSETH \\ University of Minnesota, Minneapolis, Minnesota
}

\begin{abstract}
This paper is concerned with the measurement of variation or dispersion for nominal categorical data. A new measure of such variation is proposed. It is defined as the complement of the standard deviation of the individual category frequencies from the modal frequency. Large-sample distribution methods are developed for constructing confidence intervals and testing hypotheses for the equivalent population measure of variation. Numerical sample data are used to exemplify the statistical inference methods.
\end{abstract}

For measuring the variation or dispersion in a set of nominal data, a number of alternative measures have been defined in the literature. Such measures are generally functions of the absolute frequencies or counts $n_{1}, n_{2}, \ldots$, $n_{I}$ for a set of $I$ categories that are exhaustive and mutually exclusive. Thus, among the total number of $n=\sum_{i} n_{i}$ observations (individuals or items), $n_{i}$ observations belong to category $i$, with each observation belonging to one and only one category. Among the best known variation measures for the $n_{i}(i=1,2, \ldots, I)$ are those that may be termed the mean deviation from the mode (MDM), the index of qualitative variation (IQV), and the relative entropy index (REI). These three measures may be defined as follows (e.g., Reynolds, 1977, p. 30):

$$
\begin{aligned}
\mathrm{MDM} & =1-\frac{\sum_{i}\left(n_{m}-n_{i}\right)}{n(I-1)}, \\
\mathrm{IQV} & =\left(\frac{I}{I-1}\right)\left(1-\sum_{i}\left(n_{i} / n\right)^{2}\right),
\end{aligned}
$$

and

$$
\mathrm{REI}=\frac{-\sum_{i}\left(n_{i} / n\right) \log \left(n_{i} / n\right)}{\log I},
$$

where $n_{m}$ in Equation 1 is the modal frequency, that is,

$$
n_{m}=\max \left\{n_{1}, n_{2}, \ldots, n_{1}\right\} .
$$

The summations in Equations 1 through 3 are over all $i$ from $i=1$ to $i=I$.

Each of the three measures varies between 0 and 1 . They take the value of 0 when all observations fall in a single category, and they equal 1 when equally many observations fall in all categories, irrespective of the number of categories. Consequently, these measures may be appropriate when comparing the variation in different data sets having different numbers of categories. Of the three

Address correspondence to Tarald $O$. Kvalseth, Department of Mechanical Engineering, University of Minnesota, Minneapolis, Minnesota 55455. measures, the IQV in Equation 2 appears to be the most widely accepted, especially in the social sciences (e.g., Ott, Mendenhall, \& Larson, 1978, pp. 110-113). The measure in Equation 3, which is based on Shannon's (1948) entropy in information theory, has been used quite extensively in the biological sciences (Pielou, 1977).

The measure MDM in Equation 1 suffers from one serious limitation: its value is determined exclusively by the relative modal frequency $n_{m} / n$ and $I$ (the number of categories). This fact that MDM is unaffected by frequencies other than the modal frequency is readily observed by expressing MDM differently, as done below. The purpose of the present paper is to propose an alternative measure of variation for nominal data that is based on deviations from the mode, but that depends on all the frequencies and not only the modal frequency. Furthermore, the large-sample distribution properties of the new measure are derived; this permits statistical hypotheses to be tested and confidence intervals to be constructed for this variation measure. The statistical inference procedures are exemplified using numerical data.

\section{A NEW MODAL-FREQUENCY MEASURE}

In terms of the population probabilities $p_{i}(i=1,2, \ldots$, $I)$, the population analogue of the expression in Equation 1 is

$$
\begin{aligned}
V_{1} & =1-\frac{1}{I-1} \sum_{i}\left(p_{m}-p_{i}\right) \\
& =1-\frac{I p_{m}-1}{I-1},
\end{aligned}
$$

where

$$
p_{m}=\max \left\{p_{1}, p_{2}, \ldots, p_{I}\right\} .
$$

From Equation $4 \mathrm{~b}$ it is seen that $V_{1}$ and its estimator $\hat{V}_{1}$ $=\mathrm{MDM}$, obtained by substituting the observed relative frequencies $\hat{p}_{i}=n_{i} / n$ for the $p_{i}(i=1,2, \ldots, I)$ in Equation 4 , are determined exclusively by the modal probabilities $p_{m}$ and $\hat{p}_{m}=n_{m} / n$, respectively. 
The measure $V_{1}$ is based on the first-order mean of the deviations of the $p_{i}$ from the modal probability $p_{m}$. Consider instead the following measure based on the secondorder mean of the $(I-1)$ deviations $\left(p_{i}-p_{m}\right)$ :

$$
V_{2}=1-\left\{\frac{1}{I-1} \sum_{i}\left(p_{m}-p_{i}\right)^{2}\right\}^{1 / 2} .
$$

In terms of the terminology used when measuring the variation of quantitative variables, $V_{2}$ is simply the complement of the standard deviation of the $p_{i}$ from $p_{m}$. The estimate $\left(\hat{V}_{2}\right)$ of $V_{2}$ obtained by substituting the $\hat{p}_{i}=n_{i} / n$ for all the $p_{i}$ in Equation 5 is given by the standard deviation from the mode (SDM) measure:

$$
\mathrm{SDM}=1-\left\{\frac{1}{n^{2}(I-1)} \sum_{i}\left(n_{m}-n_{i}\right)^{2}\right\}^{1 / 2} .
$$

The properties of $V_{2}$ (and hence of $\hat{V}_{2}=$ SDM) may be outlined as follows: (1) $V_{2}$ takes into account all the probabilities $p_{i}(i=1,2, \ldots, I)$ and the number of categories $I ;(2) V_{2}$ ranges in value between 0 and 1 , inclusive; (3) $V_{2}=0$ if and only if there exists an $i$ such that $p_{i}=1$ (SDM $=0$ only when all observations belong to a single category); and (4) $V_{2}=1$ if and only if $p_{i}=1 / I$ for all $i$ (SDM $=1$ only when the observations are distributed uniformly over all the categories).

The preceding properties of the proposed measure follow immediately from Equation 5 (and Equation 6). It may also be pointed out that, when comparing $V_{2}$ in Equation 5 with $V_{1}$ in Equation 4, $V_{2} \leq V_{1}$ with equality for $I=2$ (i.e., the nominal variable has two categories) or for the two extremes of no or maximum variation defined under Properties 3 and 4 above; the inequality follows from a well-known theorem for generalized means (Hardy, Littlewood, \& Pólya, 1952, Theorem 16). Properties 1-4 also hold for the measures IQV and REI in Equations 2 and 3 and for their population analogues.

\section{STATISTICAL INFERENCES}

In addition to using SDM as a descriptive statistic for the variation in the observations $n_{i}(i=1,2, \ldots, I)$, it may clearly be of interest to make statistical inferences about the underlying population quantity $V_{2}$. Thus, an investigator may want to test certain hypotheses about $V_{2}$ and construct confidence intervals. Such approximate inferences can be made by using asymptotic distribution methods, as will be demonstrated in some detail.

Under the usual multinomial sampling model with the total number of observations $\boldsymbol{n}$ fixed, it follows from the delta method (e.g., Agresti, 1984, pp. 247-250; Bishop, Fienberg, \& Holland, 1975, chap. 14) that the estimator $\hat{V}_{2}=$ SDM of $V_{2}$ is asymptotically normally distributed with mean $V_{2}$ and variance $\sigma^{2}\left(\hat{V}_{2}\right)$ that may be derived as follows. The partial derivatives of $V_{2}$ in Equation 5 with respect to the $p_{i}(i=1,2, \ldots, I)$ are given by

$$
\begin{aligned}
V_{2 i}^{\prime}=\frac{\partial V_{2}}{\partial p_{i}} & =\frac{p_{m}-p_{i}}{(I-1)\left(1-V_{2}\right)} \quad \text { for } p_{i} \neq p_{m} \\
& =\frac{1-I p_{m}}{(I-1)\left(1-V_{2}\right)} \quad \text { for } p_{i}=p_{m} .
\end{aligned}
$$

It is also easily determined that

$$
\bar{V}_{2}=\sum_{i} p_{i} V_{2 i}^{\prime}=V_{2}-1 \text {. }
$$

The variance $\sigma^{2}\left(\hat{V}_{2}\right)$ is given by

$$
\sigma^{2}\left(\hat{V}_{2}\right)=\frac{1}{n}\left(\sum_{i} p_{i}\left(V_{2 i}^{\prime}\right)^{2}-\bar{V}_{2}^{2}\right),
$$

so that when the expressions in Equations 7 and 8 are substituted into Equation 9, the following variance formula is obtained:

$$
\begin{aligned}
\sigma^{2}\left(\hat{V}_{2}\right)= & \frac{1}{n(I-1)^{2}\left(1-V_{2}\right)^{2}}\left\{\sum_{i} p_{i}\left(p_{m}-p_{i}\right)^{2}\right. \\
& \left.+p_{m}\left(1-I p_{m}\right)^{2}\right\}-\frac{\left(1-V_{2}\right)^{2}}{n}
\end{aligned}
$$

provided $V_{2}$ is neither 0 nor 1 .

When $n$ is reasonably large, and when the estimated variance $\hat{\sigma}^{2}\left(\hat{V}_{2}\right)$ is computed from Equation 10 by replacing $p_{i}$ by the observed proportions $\hat{p}_{i}=n_{i} / n$ for all $i$, approximate inferences can be made about the population measure $V_{2}$ based on the preceding results. For most practical purposes, an investigator is likely to be particularly interested in constructing a confidence interval for $V_{2}$ and testing the hypothesis that two or more $V_{2}$ values are equal. Thus, an approximate $(1-\alpha) 100 \%$ confidence interval for $V_{2}$ is given by

$$
\hat{V}_{2} \pm z_{\alpha / 2} \hat{\sigma}\left(\hat{V}_{2}\right) \text {, }
$$

where $z_{\alpha / 2}$ is obtained from the standard normal tables (e.g., $z_{\alpha / 2}=1.96$ for $\alpha=.05$ ). The null hypothesis that two $V_{2}$ values are equal, that is, $\mathrm{H}_{0}: V_{21}=V_{22}$, can be tested (against the alternative hypothesis $\mathrm{H}_{1}: V_{21} \neq V_{22}$ ) using the test statistic

$$
Z=\frac{\hat{V}_{21}-\hat{V}_{22}}{\left\{\hat{\sigma}^{2}\left(\hat{V}_{21}\right)+\hat{\sigma}^{2}\left(\hat{V}_{22}\right)\right\}^{1 / 2}}
$$

and rejecting $\mathrm{H}_{\mathrm{o}}$ at the $\alpha$ level of significance if $|Z|>Z_{\alpha / 2}$. This testing procedure is based on the assumption that the estimators $\hat{V}_{21}$ and $\hat{V}_{22}$ are independent, that is, that they are based on two independent samples.

Consider now the more general hypothesis $\mathrm{H}_{\mathrm{o}}: V_{\mathbf{2 1}}=$ $V_{22}=\ldots=V_{2 k}$ for $k \geq 2$. Define the weighted average of the $\hat{V}_{2 j}(j=1,2, \ldots, k)$ as

$$
\overline{\hat{V}}_{2}=\sum_{j} w_{j} \hat{V}_{2 j} / \sum_{j} w_{j} ; w_{j}=1 / \hat{\sigma}^{2}\left(\hat{V}_{2 j}\right) \text {. }
$$

If the samples of sizes $n_{j}(j=1,2, \ldots, k)$ are indepen- 
dent and reasonably large, the hypothesis of equal variations may be tested by referring the value of the statistic

$$
\chi^{2}=\sum_{j} \frac{\left(\hat{V}_{2 j}-\overline{\hat{V}}_{2}\right)^{2}}{\hat{\sigma}^{2}\left(\hat{V}_{2 j}\right)}
$$

to tables of the chi-square distribution with $k-1$ degrees of freedom (e.g., Agresti, 1984, pp. 190-194; Fleiss, 1981 , section 10.1). The hypothesis is rejected at the $\alpha$ level of significance if the value of Equation 14 exceeds the upper $100 \alpha \%$ point of the chi-square distribution with $k-1$ degrees of freedom. If the null hypothesis is rejected, pairwise comparisons can be made using Equation 12. If the null hypothesis is not rejected, so that the population variations $V_{2 j}$ can reasonably be assumed to be equal for $j=1,2, \ldots, k$, then $\hat{V}_{2}$ in Equation 13 may serve as an overall variation estimate.

\section{NUMERICAL EXAMPLES}

To demonstrate the use of the variation measure SDM and the statistical inference methods developed above, some numerical sample data have been used. Consider first the case when a sample of size $n=150$ is distributed across $I=3$ nominal categories as follows: $n_{1}=75, n_{2}=67$ and $n_{3}=8$. These $n_{i}$ may, for example, be individuals classified as Democrat, Republican, or Independent. The variation in these data is computed from Equation 6 as being

$$
\begin{aligned}
& \operatorname{SDM}= \\
& 1-\left\{\frac{1}{(150)^{2}(3-1)}\left[(75-67)^{2}+(75-8)^{2}\right]\right\}^{1 / 2}=.68 .
\end{aligned}
$$

By comparison, $\mathrm{MDM}=.75$ from Equation 1 or MDM $=\hat{V}_{1}=.75$ from Equation $4 \mathrm{~b}$ with $\hat{p}_{m}=75 / 150$. From Equations 2 and $3, \mathrm{IQV}=.82$ and $\mathrm{REI}=.79$.

By substituting $\hat{p}_{i}=n_{i} / n$ for $p_{i}$ and $\hat{V}_{2}=\mathrm{SDM}=.6819$ for $V_{2}$ in Equation 10, the estimated variance is computed as $\hat{\sigma}^{2}\left(\hat{V}_{2}\right)=.0016$. According to Equation 11, an approximate $95 \%$ confidence interval for the population measure $V_{2}$ is given by $.6819 \pm 1.96(.0016)^{1 / 2}$, or $(.60, .76)$.

Consider next that the previous data referred to the party affiliation of a sample of males, whereas another independent sample of females resulted in $n_{1}=114, n_{2}=76$, and $n_{3}=10$. Based on these data and Equations 6 and 10, it is found that $\mathrm{SDM}=\hat{V}_{22}=.6085$ and $\hat{\sigma}^{2}\left(\hat{V}_{22}\right)=.0018$ as compared with the previous values of $\hat{V}_{21}=.6819$ and $\hat{\sigma}^{2}\left(\hat{V}_{21}\right)=.0016$ for males. When these results are substituted into Equation 12, the value of the test statistic becomes $Z=1.26$ so that $\mathrm{H}_{0}: V_{21}=V_{22}$ is not rejected (at the .05 level of significance since $1.26<Z_{\alpha / 2}=1.96$ for $\alpha=.05)$. Consequently, it is concluded that the variation in political party affiliation does not differ significantly between males and females.

Finally, consider that the preceding two data sets refer to married males and females. Two additional indepen- dent samples of unmarried males and females produced $m_{i}=110,80,10$ and $n_{i}=135,120,45$. For these data sets 3 and 4 , it is found that $\hat{V}_{23}=.6309, \hat{\sigma}^{2}\left(\hat{V}_{23}\right)=.0016$, $\hat{V}_{24}=.7849$, and $\hat{\sigma}^{2}\left(\hat{V}_{24}\right)=.0011$. When these results together with those of the previous two data sets are substituted into Equations 13 and 14 , it is found that $\hat{\hat{V}}_{2}=$ .6896 and $\chi^{2}=14.10$. Since 14.10 exceeds even the tabulated chi-square value of 12.84 for $\alpha=.005$ and $k-1$ $=3$ degrees of freedom, the decision is to reject $\mathrm{H}_{0}: V_{21}$ $=V_{22}=V_{23}=V_{24}$. Thus, it is concluded that the variation in party affiliation differs significantly $(p<.005)$ between the four population groups. It has already been decided that no such significant difference exists between married males and females, so that additional pairwise comparisons using Equation 12 are needed if the specific sources of the variation difference are to be determined.

\section{CONCLUDING COMMENTS}

In the preceding numerical examples, the same number of categories $(I=3)$ were used when comparing the population $V_{2 j}$ for different $j$. However, the same statistical procedures apply if the number of categories $\left(I_{j}\right)$ differs for different $j$. Similarly, the sample measure or descriptive statistic SDM is appropriate for comparing the variation in data sets with differing number of categories since SDM takes into account the number of categories.

The new measure SDM is proposed as being suitable and useful for measuring the variation or dispersion in nominal data, that is, when the underlying variable is measured on a nominal scale. It is not a proper measure, nor are the alternative measures in Equations 1-3 appropriate, when the data or the underlying variable is ordinal, since SDM does not use the ordering among the categories. In fact, if SDM or the other measures in Equations 1-3 are used for ordinal categorical data, misleading results may occur. For measuring the variation in ordinal data, an index such as that proposed by Leik (1966) is more appropriate.

In conclusion, the SDM measure and the associated statistical inference methods presented in this paper offer alternative means of analyzing the variation for nominal variables. Although SDM is clearly preferable to MDM in Equation 1, the desirable properties of SDM are also possessed by IQV and REI in Equations 2 and 3. Thus, the choice of measure to use in any given situation is up to the individual investigator. The use of more than one variation measure for each nominal data set is perhaps the compromise and most prudent solution.

\section{REFERENCES}

AGRESTI, A. (1984). Analysis of ordinal categorical data. New York: Wiley.

Bishop, Y. M. M., Fienberg, S. E., \& Holland, P. W. (1975). Discrete multivariate analysis: Theory and practice. Cambridge, MA: MIT Press. 
FLEISS, J. L. (1981). Statistical methods for rates and proportions. New York: Wiley.

Hardy, G. H., Littlewood, J. E., \& Pólya, G. (1952). Inequalities (2nd ed.). Cambridge, England: Cambridge University Press.

Leik, R. L. (1966). A measure of ordinal consensus. Pacific Sociological Review, 9, 85-90.

Ott, L., Mendenhall, W., \& Larson, R. F. (1978). Statistics: A tool for the social sciences. North Scituate, MA: Duxbury.
Pielou, E. C. (1977). Mathematical ecology. New York: Wiley. REYNOLDS, H. T. (1977). The analysis of cross-classifications. New York: Free Press.

Shannon, C. E. (1948). A mathematical theory of communication. Bell System Technical Journal, 27, 379-423, 623-656.

(Manuscript received for publication January 30, 1988.) 